\title{
CIEGO DE ÁVILA (CUBA): RETROSPECTIVA ESCÉNICA. EL TEATRO IRIONDO
}

\author{
Gerardo Fuentes Pérez \\ Real Academia Canaria de Bellas Artes de San Miguel Arcángel
}

\section{RESUMEN}

Por su situación geográfica, Ciego de Ávila fue un nudo de comunicaciones entre el oriente y el occidente de Cuba, logrando una prosperidad económica gracias al impulso de la industria azucarera a mediados del siglo XIX. Es a partir de este momento cuando la actividad teatral se va a convertir en una de las principales necesidades culturales de aquella población pujante que convirtió el arte de la escena en su propia propaganda social, de modo que promovieron la creación de compañías y grupos locales, logrando con ello convertirse en uno de los espacios teatrales más importantes de la isla; el Iriondo y el Principal son los locales de mayor actividad teatral y cinematográfica.

Palabras Clave: ciego, corral de comedias, compañía teatral.

\section{CIEGO DE ÁVILA (CUBA): SCENIC RETROSPECTIVE. IRIONDO THEATRE}

\section{Abstract}

Due to its geographical location, Ciego de Avila was a communicative point between the East and the West of Cuba, achieving economic prosperity thanks to the increase of the sugar industry in the mid-19th century. From this time on, the theatrical activity is going to become one of the main cultural needs of that thriving population that used artistic activities as their own social propaganda. With this action, they promoted the creation of local companies and theatrical groups, which made of Ciego de Avila one of the most important theatrical spaces in Cuba; the Iriondo and the Principal are the places where most theatrical and cinematographic activities took place.

Keywords: Ciego, Corral of comedies, Theatre Company. 
No es nuestra intención dar a conocer la historia de esta ciudad de Cuba, suficientemente estudiada por autorizados investigadores y estudiosos locales y foráneos. Pero bien es cierto que su origen y su discurrir a través de los años nos permiten acercarnos al aspecto cultural y, más concretamente, a su producción teatral. Su mismo nombre lo indica: «Ciego» es una acepción lingüística muy peculiar en aquella isla caribeña, refiriéndose a un terreno cultivado en medio de los claros de los bosques, equivalente al término "hato", muy usado en el castellano de Sudamérica $^{1}$. Fue en 1538 cuando Jácome de Ávila recibe tierras en este lugar. El «ciego» reunía una serie de viviendas (ranchos) de trabajadores que terminaron por convertirse en una próspera población.

Por su situación geográfica, Ciego de Ávila fue un nudo de comunicaciones entre el oriente y el occidente de Cuba, logrando un florecimiento económico gracias al impulso de la industria azucarera a mediados del siglo XIx. Sin embargo, en la centuria anterior ya había logrado una serie de prerrogativas que la llevarían a conseguir la categoría de ciudad, y más tarde capital de su provincia. Nos referimos a la creación de la Capitanía pedánea en 1764 y a una serie de establecimientos como, por ejemplo, las oficinas de Correos. La industria azucarera fue la que consolidó la economía de la ciudad y de toda su comarca, atrayendo a numerosos industriales, comerciantes y gente que buscaba mejores rentas al calor del capital, de los cultivos, trapiches e ingenios, destacando los de Soledad, cuyo propietario fue José María Iznaga del Valle ${ }^{2}$ y Resurrección, regentado por el conde de Villamar. El crecimiento de la población trajo consigo que durante la segunda mitad del siglo XIX esta ciudad de Ciego de Ávila conociera profundas remodelaciones urbanas, estableciéndose un planeamiento cuadricular, en el que se organizaban las viviendas de una o dos plantas, generalmente con soportales, a la manera de alpendres, cuya estructura y diseño dependía de la capacidad económica de cada una de las familias. Así, se levantaron columnas de carácter clásico, a veces de estilo ecléctico, con formas caprichosas, sobresaliendo los distintos colores aplicados a cada una de las partes que las componen; otras, en cambio, ofrecen soluciones más modestas, sin demasiadas pretensiones, todo ello resuelto con madera, de tradición hispana. Se esta-

${ }^{1}$ Con el tiempo, estos «ciegos» fueron perdiendo su espacio original a medida que se convertían en poblaciones organizadas y estables. En Cuba aún se mantiene este topónimo a lo largo de toda su geografía, aunque la mayor parte de ellos se concentran en la zona centro y en toda la región de Camagüey; Ciego de Escobar, Ciego de San Antonio, Ciego de Najasa, Ciego de Curana, Ciego de Abajo, Ciego Montero, Ciego Largo, etc., son ejemplos de la actividad agropecuaria.

2 Apellido de origen vasco. El primero en llegar a Cuba fue Antonio Damián de Iznaga y Albiz (Bilbao, 1696-Bayona, 1731). Contrajo matrimonio en La Habana con Catalina Villa. Uno de los descendientes, José María Iznaga del Valle (1819-1892), disfrutó de grandes extensiones de tierras en Ciego de Ávila. Estuvo casado con Petronila García y Echemendia, con quien procreó a José Antonio y a Antonio Modesto Iznaga García. El segundo, propietario del ingenio Resurrección fue José Fernando Hernández y Socarrás, IV conde de Villamar. El primero en ostentar este título nobiliario, concedido por el rey Fernando VII (1816), fue Santiago Hernández Rivadeneira, natural de la ciudad de Zamora, originario de La Laguna, en Tenerife. Fue alcalde mayor de la entonces villa y Puerto Príncipe (hoy Camagüey). 
blecieron las escuelas públicas, la línea telegráfica, el local de la prensa, farmacias, centro de enseñanzas musicales, etc., aparte de la aparición de las habituales sociedades de recreo, como "La Popular», y el trazado ferroviario. A finales del citado siglo ya se habían levantado algunos de los edificios más representativos, como los actuales museos de la Historia (1874) y de Artes Decorativas (1899) y el Ayuntamiento (1877), aparte de construcciones particulares, de fachadas singularizadas, amplios zaguanes, patio central, jardines y huertas. Es entonces cuando el teatro irrumpe con fuerza, convirtiéndose en el primer espectáculo público, siendo considerado y protegido por la casta política, que estimuló la redacción de planes y programas para el desarrollo de las enseñanzas escénicas, de modo que en 1846 tuvo lugar la creación de la primera compañía teatral. La aparición de grupos con clara vocación escénica logró que Ciego de Ávila llegara a constituirse en uno de los espacios teatrales más importantes de Cuba.

$\mathrm{Al}$ principio, tal y como sucedía en la metrópolis, el teatro se desarrollaba en las casas particulares aprovechando las estancias más amplias, así como los patios centrales, a la manera de los antiguos corrales de comedia. El edificio donde tuvo la sede la Colonia Española (hoy Casa de la Cultura) improvisaba con frecuencia un escenario para las representaciones escénicas. También en la que fue sede de la Sociedad de Instrucción y Recreo La Popular (actual Museo de Artes Decorativas). Este interés por el teatro hizo necesaria la construcción de locales independientes destinados a estas funciones impulsados por los máximos agentes sociales, que dispusieron de interesantes colecciones bibliográficas ${ }^{3}$, con numerosos títulos de asuntos teatrales que traían del extranjero, sobre todo de España ${ }^{4}$. En general, disponían de una amplia cultura, actuaban como verdaderos filántropos, dominaban las artes del comercio y solían hablar varias lenguas. Su afición al teatro les permitía ser ellos mismos los principales actores, independientemente de aquellos vecinos invitados a participar en la puesta en escena. No cabe duda de que el teatro supuso una manera de potenciar el poder social de la burguesía urbana. No hemos encontrado informaciones que nos puedan aportar datos acerca de la composición de las escenografías, de los materiales utilizados, de los recursos luminotécnicos, de los mecanismos que hicieron posible la aparición de los decorados, personajes, etc. Sin embargo, la instalación de todas las tramoyas obedecía siempre al mismo patrón, pues se elegía tanto el fondo de los patios como el del salón principal para ello, de modo que se aprovechaban las puertas y ventanas como decorados, utilizándose además la escalera. La actuación en el patio solía tener un carácter más abierto y admitía al público transeúnte al que se agasajaba con licores y dulces. Pero el espectáculo de los salo-

${ }^{3}$ Citemos a manera de ejemplo la surtida y amplia biblioteca del intelectual avileño Antonio Benedicto Rodríguez (1882-1947), una de las mejores de toda la región.

${ }^{4}$ Uno de tantos ejemplos lo encontramos en el Puerto de la Cruz (Tenerife), en la persona de don Bernardo Cólogan Fallon (1772-1814), destacado comerciante y filántropo. Dispuso de una excelente colección de obras de teatro (españolas, inglesas y francesas) que solían interpretar tanto en su casa de La Paz como en su establecimiento de la actual calle Quintana (hoy Hotel Marquesa) de la citada ciudad tinerfeña. Los actores eran miembros de su familia y vecinos aficionados. 


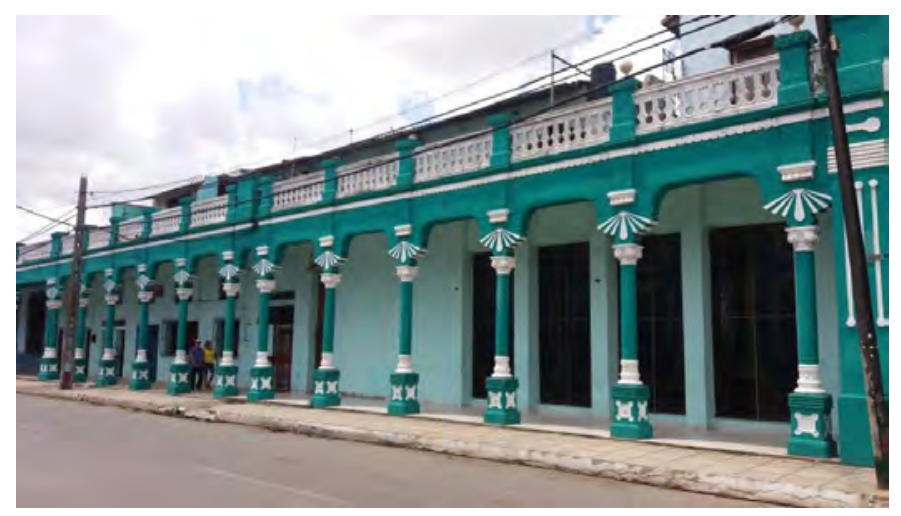

Foto 1. Ciego de Ávila (Cuba). Soportales de la calle.

nes estaba reservado para la familia e invitados elegidos. La presencia de los soportales en las fachadas ${ }^{5}$ permitía la acogida del público en los continuos días de calor caribeño o en momentos de atronadoras lluvias.

Uno de esos próceres que apoyaban y patrocinaban el teatro fue don Vicente Iriondo de la Vara (1863-1963), un asturiano nacido en Oviedo y que después de haber cursado estudios medios, emigró a Cuba cuando solo contaba 19 años de edad en busca de nuevas perspectivas de bienestar. A mediados del siglo XIx, la colonia asturiana en aquella isla era ya muy significativa, creciendo como organización social, económica y cultural a lo largo de la citada centuria, dedicada básicamente al comercio, a la industria, banca y explotación azucarera. Los asturianos se extendieron por toda Cuba, concentrándose sobre todo en La Habana, Matanzas, Pinar del Río, Cienfuegos y Santa Clara ${ }^{6}$. Don Vicente residió en la capital, empleándose como tenedor de libros y cajero en la fábrica de cigarros de Pedro Antonio Estanillo y Trueba, quien llegó a controlar un grupo de marcas de tabaco en esos años?.

5 A Ciego de Ávila se le conoce también como La ciudad de los soportales. No hay en esta ciudad un rincón, un espacio ni un resquicio en el que no haya soportales. El efecto estético y visual es extraordinario, a pesar del evidente abandono de estas viviendas por falta de recursos económicos y de una gestión política adecuada. Los variados colores, las texturas y la variedad de formas producen un resultado arquitectónico y urbano de primer orden. Estos soportales adquieren un protagonismo único, convirtiéndose en la verdadera "fachada» de los edificios, produciendo muchas veces auténticos túneles a lo largo del infinito recorrido de sus calles. Hay que indicar que la mayoría de estas edificaciones eran -y son- de una sola planta, por lo que los vanos de la fachada (puerta central como eje divisorio y ventanas laterales) quedaban bajo la protección de estos soportales.

${ }^{6}$ Para conocer este amplio tema migratorio es obligatorio consultar los estudios de Enrique Collazo Pérez, Eddy Vargas, Cecilia Hernández Rodríguez, Roberto Rivacoba Cachón, Ángel Cabrera, Álvaro Armengol, Magaly Zamora y Silvia Cherem, entre otros.

7 Su área de exportación fue realmente amplia, abarcando prácticamente todo el Caribe y buena parte de América del Sur. La mercancía llegó a situarse en Colombia, teniendo como foco 
Fue un hombre autodidacta, con una gran cultura, poseedor de una amplia biblioteca e interesado por temas curiosos, siempre en relación con el mundo moderno, la naturaleza, el cosmos, como si de un futurista se tratara, admirando el tiempo, la energía, la fuerza, lo oculto. Se interesó por el funcionamiento de la electricidad y, de una manera especial, por el cine. Uno de sus títulos, El mecanismo del Universo, publicado en cuba en 1924, lo pone de manifiesto. También escribió Las Las Leyes de la Repulsión Universal y la Evolución de los Mundos, Precogniciones, esta última una interesante y amena autobiografía. Cultivó la poesía y múltiples ensayos.

Su literatura permite obtener una visión más o menos amplia de la situación histórica y personal de entonces. Tuvo una vida holgada, aunque al final de sus días ya la ceguera le había impedido ver. Cuentan sus biógrafos que su fortuna se acrecentó gracias a la obtención del máximo premio en el sorteo de la lotería (1890), por lo que sus empresas consiguieron una estabilidad garantizada, contando con negocios en Ciego de Ávila que en aquellos tiempos de abundancia, marchaban próspera$m e n t e^{8}$. Entre sus muchos establecimientos regentó, junto con su hermano Manuel, un aserradero en la finca llamada "Colonias», a seis kilómetros de la mencionada ciudad, con aparatos modernisimos, movidos a vapor ${ }^{9}$. En 1895, en plena Guerra de la Independencia, la finca disponía de unos 50 bueyes destinados al trasporte de la madera hasta el puerto Júcaro, al sur de la provincia. Fue también propietario de una central azucarera. A pesar de haber sido considerado una persona de notoria reputación, la hacienda "Colonias» se vio de vez en cuando "visitada» con nocturnidad y alevosía por asaltadores, lo que le obligó a montar guardia en todo su territorio; Duque, el perro, fue un eficaz colaborador tanto para repeler como para descubrir la identidad de los delincuentes.

Sin embargo, como pensador y filántropo, don Vicente Iriondo invirtió en cultura, impulsando los espectáculos artísticos en Ciego de Ávila, una ciudad que crecía, haciéndose necesarios locales apropiados para las artes escénicas. Así surgió la idea de construir un teatro a la medida de las posibilidades y exigencias de la población. Y así nació el popular teatro-cine Iriondo, cuyos trabajos comenzaron el 8 de noviembre de 1909, para abrir sus puertas al año siguiente.

No disponemos de noticias relativas a la compra de terrenos, materiales y demás para conocer los principios de la construcción de este edificio. Lo cierto es que fue todo un acontecimiento para la ciudad, en un momento en que el cine comenzaba a despuntar como un espectáculo seguro y de masas, de modo que don Vicente Iriondo ya auguraba un rentable negocio. El arquitecto fue Pedro de Pastors Martínez de Villa, quien había llevado a cabo otros proyectos en esta ciudad, como la Casa de la Cultura, levantada en 1907 para albergar la Colonia Española.

principal la ciudad de Medellín. Consultar a OchoA, Lisandro: Cosas viejas de la Villa de la Candelaria. Colección Biblioteca Básica de Medellín (Colombia), n.o 4 (ITM), Medellín, 2004, p. 90.

${ }^{8}$ Alfonso Fernández, Adalberto: Mis investigaciones... y algo más. Obras completas, tomo I, Ed. Palibrio, Madrid, 2011, p. 361.

9 Idem, p. 323. 


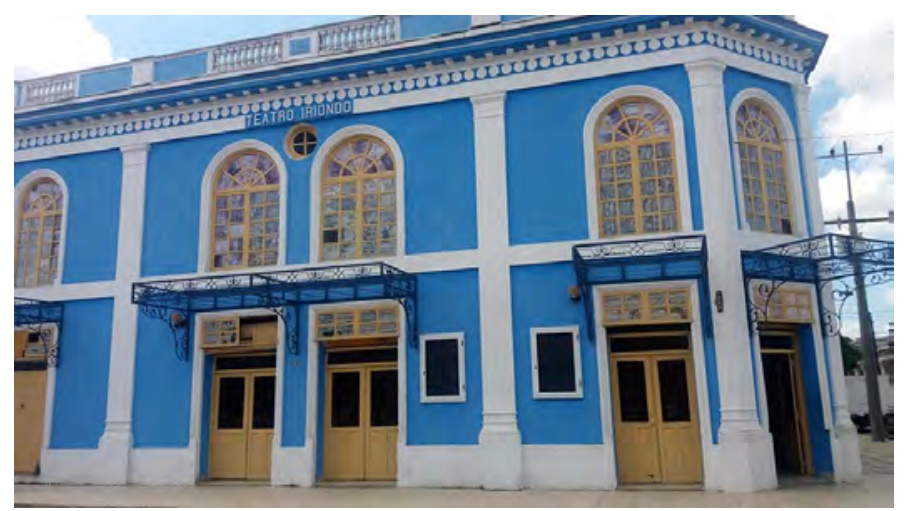

Foto 2. Teatro Iriondo. Ciego de Ávila (Cuba).

Teniendo en cuenta que el futuro edificio cumpliría la función de sala cinematográfica, Pedro de Pastors lo planteó de forma rectangular, prescindiendo de soluciones a la italiana. Este recurso era ya muy habitual en muchas salas destinadas a cumplir esta doble función: teatro-cine. El sentido longitudinal de la planta permite contemplar la pantalla en toda su amplitud, pues la imagen proyectada siempre está en un primer plano, mientras que una representación teatral requiere muchos ángulos debido a la perspectiva, dirección y a la propia acción de la obra.

El edificio, que abrió sus puertas el 1 de agosto de 1910, era algo más simple que el que vemos hoy. La instalación de la cabina de proyecciones (1922) fue una de las causantes de la transformación del interior, que se concibe de dos plantas, convirtiéndose la superior en el verdadero palco, que recorre ininterrumpidamente todo el trayecto a ambos lados del patio de butacas, no existiendo una separación con respecto al llamado "gallinero». Estos palcos se apoyan en sencillos pilares de capiteles moldurados. Ante la simpleza decorativa, destaca el escenario, de 20 metros de ancho, cuya embocadura la constituyen elementos arquitectónicos sin demasiadas pretensiones dentro de una indiscutible sobriedad. El exterior, en cambio, ofrece un cierto encanto por la distribución de los vanos entre pilastras de soluciones clásicas y remate con balaustradas, originando así un eclecticismo bastante equilibrado. En su origen, esta fachada seguía los esquemas de la arquitectura tradicional del lugar, es decir, con un alargado soportal, que más tarde fue eliminado.

Comenta la prensa del momento que asistió al acto de inauguración el alcalde de la ciudad, don Adolfo Morgado Paz (+1926), y que después de las palabras protocolarias, se disfrutó de un concierto a cargo de la Banda Infantil. Al finalizar se proyectaron cuatro películas de corta duración y se representarán las zarzuelas $E l$ diablo con faldas (Ruperto Chapí, 1908) y El cabo primero (Manuel Fernández Caballero, 1895), por la compañía de Manolo Vázquez.

A lo largo de esta primera etapa, el escenario del Iriondo acogió a conocidos artistas tanto del ámbito teatral como de la canción, entre los que destacamos a Arquímedes Pous (1891-1926), el popular «negrito» del teatro vernáculo cubano, 


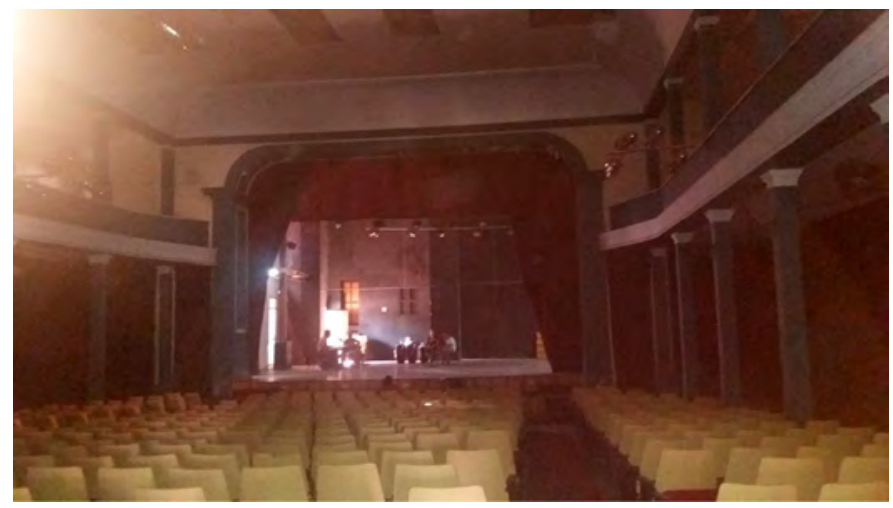

Foto 3. Teatro Iriondo. Interior. Ciego de Ávila (Cuba).

que aparte de haber sido un relevante actor, también demostró ser un excelente coreógrafo y dramaturgo; Enrique Arredondo (1906-1988), actor de teatro, cine y televisión; Candita Quintana (1912-1977), consagrada al teatro y televisión; Esperanza Iris (1888-1962), una de las grandes divas del teatro mexicano, cuya actuación se produjo el 24 de junio de 1914; Jorge Negrete, destacado cantante y actor mexicano; la conocida actriz y cantante argentina Libertad Lamarque (1908-2000), y nada menos que la célebre actriz francesa Sarah Bernhardt (1844-1923), que recorrió toda América a partir de 1879. Frisaba ya los 70 años de edad cuando cautivó a todo el público de Ciego de Ávila en este Teatro Iriondo. Entre las principales actuaciones, hay que destacar, finalmente, la ofrecida por la Compañía de Títeres de Vittorio Podrecca (1883-1959), de Roma, en 1925. Impresionó al público por la aparatosidad de las escenas, la acción, la variedad musical y los numerosos muñecos (títeres), que superaban los 2000 ejemplares. Aparte de estas grandes figuras, eran frecuentes las actuaciones de compañías y grupos locales, aunque la actividad cinematográfica ganaba adeptos y espacios.

Parece que hay acuerdo entre los historiadores en asegurar que fue el Teatro Iriondo el primero en dar a conocer el arte cinematográfico. Desde el punto de vista funcional, hay que reconocerlo. Pero unos ańos antes, en 1908, una empresa mexicana ya proyectaba películas con un biscopio Pathé en el edificio de la Colonia Española.

Hemos dicho que en la década de los años veinte el teatro sufrió una notable transformación cuyos trabajos fueron esperados con mucho interés por parte de los habitantes de la ciudad, pues consideraban que el local debía ser debidamente moderno y con el confort y la comodidad que merece nuestra sociedad distinguida ${ }^{10}$. El

${ }^{10}$ Periódico La Región. Interdiario Comercial y de Información. Órgano de la Cámara de Comercio. Ciego de Ávila, Cuba, 28 de mayo de 1921, n. 296. 
6 de septiembre de 1922, el Iriondo reabrió sus puertas con un acto brillantísimo. Aparte de las autoridades pertinentes, participó la Banda Municipal, creada en 1914. Se proyectó una película (¿silente?) cuya protagonista era la actriz estadounidense Agnes Ayres (1898-1940). Por la noche, La herencia del suicida, con William Duncan (1879-1961), y Miel silvestre, interpretada por Prescilla Dean (1896-1987).

A partir del mencionado ańo de 1922, momento en que el cine irrumpe con fuerza en la sociedad avileña, las representaciones teatrales se redujeron considerablemente; sin embargo, el Iriondo siguió abriendo las puertas a las grandes companías que desearon actuar en su escenario, siempre y cuando los medios económicos lo permitieran, pues no resultaba nada costoso hacer escala en Ciego de Ávila, ya que era de parada obligada para cualquier empresa teatral en su periplo por la zona oriental, hacia Camagüey, Santiago de Cuba, Holguín, etc. Una de esas compañías fue la de Prudencia Grifell (1876-1970), que actuó el día 16 de agosto de $1923^{11}$. En 1917 ya había formado su propia compañía, en la que debutó la actriz mexicana Esperanza Iris. No era la primera vez que visitaba el Iriondo; el público la había admirado en distintas ocasiones... y positivamente su nombre no figurará en Compañias de poca monta ${ }^{12}$. El elenco estaba constituido por ocho actores, nueve actrices (una de ellas, su hija Amparo M. Griffel), dos apuntadores y el maquinista. La empresa teatral manifestó su orgullo de haber adquirido esta pequeña temporada de la Grifell, que complació a Ciego de Ávila con su amplio repertorio de obras de autores extranjeros y españoles ${ }^{13}$. La actriz siguió con la gira por las ciudades del oriente cubano. El éxito fue tan grande en este Teatro Iriondo que de regreso a $\mathrm{La}$ Habana volvió a detenerse en Ciego de Ávila para repetir actuación, dejando a su paso una estela radiante de simpatía ${ }^{14}$. La prensa anima a sus lectores a que adquieran cuanto antes las localidades por la constante demanda.

Los ańos 20 y 30 del mencionado siglo $\mathrm{xx}$ fueron realmente exitosos, pues tanto el Teatro Iriondo como el París Garden, el Martí, etc., incluso el Teatro Principal levantado en 1927, recibieron grandes compañías de teatro, óperas, zarzuelas, espectáculos de variedades, aparte de la actividad cinematográfica. Una ciudad como Ciego de Ávila, que no superaba los 10000 habitantes, contaba entonces con unos seis teatros, lo que indica el talante cultural y su gran afición por las artes escénicas ${ }^{15}$.

Y por el Iriondo pasaron compañías de enorme calado artístico. El 23 de agosto, por ejemplo, estuvo la Telmo Montalt, con el Genio alegre de los hermanos Serafín (1871-1938) y Joaquín (1873-1944) Álvarez Quintero. Tuvieron una excelente acogida, cosechando muchos aplausos, cuya actuación «fue inmejorable, espe-

${ }^{11}$ Periódico La Región. Interdiario Comercial y de Información. Órgano de la Cámara de Comercio. Ciego de Ávila. Cuba. Sábado, 11 de agosto de 1923, año 4, n. 219.

12 Idem: sábado, 11 de agosto de 1923, n. $^{\circ} 219$.

${ }_{13}$ Idem: entre su amplio repertorio podemos citar las siguientes obras: Cristalina, La mala ley, Embrujamiento, Remedios heroicos, Madrigal de la cumbre, El caudal de los hijos, El tiempo de las cerezas, Madre, Los malcasados.

${ }_{14}$ Idem: viernes, 24 de agosto de 1923, n. ${ }^{\circ} 229$.

15 Idem: sábado, 11 de agosto de 1923, n. $^{\circ} 219$. 
cialmente José Telmo y Antonio Montalt que en sus roles de Lucio y Julio, respectivamente, quedaron a la altura de su merecida fama. El periódico local La Región elogia la interpretación del resto de los actores, así la Sra. Pilar Fernández interpretando la vivaracha y alegre Asunción, también nos agradó sobremanera, puso en su gesto expresiones tan sublimes de alegría que no se puede por menos que aplaudir efusivamente. Los demás, desempeñaron sus papeles admirablemente. Para todos las flores más fragantes del jardín de mi admiración. Hoy se despiden de nuestro público con la colosal obra Amor Tardío»" ${ }^{16}$ de Manuel Machado (1874-1947).

Sin embargo, la compañía más esperada y que cosechaba siempre grandes éxitos fue sin duda la de Esperanza Iris, un icono de la escena. No solo fue actriz, sino también cantante y vedette. Nacida en México en 1884, representó en los principales teatros del mundo con enorme éxito. Antes de construirse el Iriondo, ya Esperanza había hecho gira por Cuba, actuando entre 1910 y 1911, aunque en 1914, recién inaugurado el mencionado local, debutó por primera vez. En Espańa interpretó en varios teatros como el Cómico y Zarzuela (Madrid) y el Apolo (Valencia), en 1922 con la obra La Princesa de las Czardas, opereta del compositor austrohúngaro Emmerich Kalman (1882-1953). Condecorada por el rey Alfonso XIII, fue también llevada al lienzo por el pintor Joaquín Sorolla (1863-1923)

En el Teatro Iriondo debutó en marzo de 1924. Fue un acontecimiento cultural y social de primer orden, pues las opiniones vertidas por la prensa fueron desbordantes y halagadoras. A la función asistió «lo más selecto y distinguido de nuestra culta sociedad avileña [...] sus palcos todos estaban adornados por encantadoras y respetables damas de nuestro mundo elegante» ${ }^{18}$ que pudieron disfrutar de Benamor, una opereta en tres actos de Pablo Luna (1879-1942), y que la propia Esperanza Iris había interpretado el 12 de mayo de 1923 en el Teatro de la Zarzuela, durante su estancia en la capital de España. Entre otros espectáculos conviene citar la zarzuela La moza de campanillas, de los maestros Antonio Paso, Ricardo González del Toro y Pablo Luna.

La compañía abandona Ciego de Ávila para continuar su periplo por las provincias orientales de la isla. En su primer viaje estuvo invitada a la inauguración del teatro Luisa (Luisa Martínez Casado), de la ciudad de Cienfuegos, en 1911, con la opereta La Viuda Alegre, del compositor austrohúngaro Franz Lehár (1870-1948). Una vez finalizada aquella serie de actuaciones por los teatros de Camagüey, Santiago de Cuba, etc., regresa a Ciego de Ávila para cumplir con el contrato que había firmado con la empresa Devesa Aragón, encargada de gestionar el Teatro Iriondo. En esta ocasión satisfizo al público avileño, a partir del 5 de mayo de 1924, con la

16 Idem: viernes, 24 agosto de 1923, n. $^{\circ} 229$.

${ }_{17}$ Este pintor valenciano realizó dos retratos de Esperanza Iris. Uno, a través de fotografías, pues jamás estuvo en México, y otro, en Valencia, durante su estancia teatral. Posó en el propio estudio del citado pintor. También se cuenta con varias fotografías del momento. Ambos lienzos se hallan en paradero desconocido. Tenemos noticias de su existencia gracias a las aportaciones gráficas.

${ }_{18}$ Periódico La Región..., viernes, 19 de marzo de 1924, n. ${ }^{\circ} 108$. 


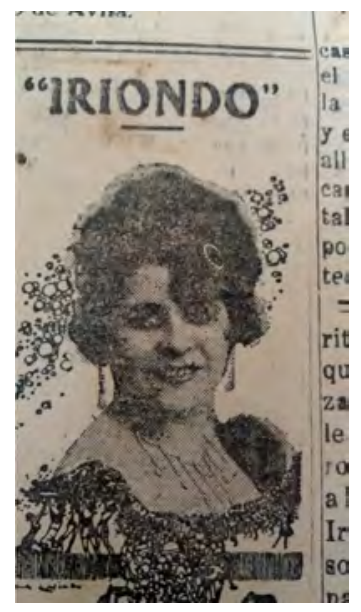

Foto 4. Esperanza Iris. Periódico La Región (Ciego de Ávila) 17-III-1924.

opereta Dedé, del maestro Christiné (1867-1941), y con la zarzuela del compositor Amadeo vives (1871-1932) titulada La gatita blanca ${ }^{19}$.

El referido teatro seguía compartiendo la actividad cinematográfica con la escénica. Con respecto a la primera, el público manifestó con frecuencia su malestar por las películas elegidas, que producían un cierto hastío bastante increíble, pretendiéndose recuperar el entusiasmo por el séptimo arte invitando a ver el film titulado El Jorobado de Nuestra Señora de París, realizado por el director Wallace Worsley $(1871-1932)^{20}$. El teatro, las actuaciones musicales, la danza, etc., aún mantenían la atención del público, que demandaba espectáculos de calidad. Así, en octubre de 1924, aprovechando la actuación de la tonadillera y bailadora española Amalia Molina (1881-1956) en La Habana, dentro de la tournée por Sudamérica, la empresa del Iriondo la contrata para que actúe el sábado 25 de octubre de la mencionada temporada. El periódico La Región se deshace en elogios y aplausos definiéndola como la «embajadora del alma espańola... la artista maravillosa que han aclamado los más aristocráticos públicos del mundo... Hay que oírla y hay que verla... Su espectáculo ha de ser un sorprendente acontecimiento ${ }^{21}$. La despedida de esta bailadora supuso una manifestación de cariño, de afectos y de sentimientos expresados en inacabables aplausos, flores y vítores.

En muchas de las actuaciones siguientes, ya se percibía una notable reducción del número de espectadores. La prensa lamentaba muchas veces ese vacío por parte del público, que solo llegaba a ocupar la mitad de las plazas. Dentro de los debuts

19 Idem: sábado, 3 de mayo de 1924, n. ${ }^{\circ} 214$.

20 Idem: miércoles, 21 de mayo de 1924, n. 226.

${ }^{21}$ Idem: jueves, 23 de octubre de 1924, n. ${ }^{\circ} 4$. 
que tuvieron lugar en el Iriondo en esta temporada conviene recordar la actuación de Eva Canel (1857-1932), la actriz española que viajó por Sudamérica ejerciendo, junto a su marido Eloy Perillán, una intensa actividad periodística y teatral. En el Iriondo interpreta la obras El indiano, La mulata y La abuelita con su elenco entre el 10 y 12 abril de $1926^{22}$.

El Teatro Iriondo languidece en cuanto a la puesta en escena se refiere. Entre las razones de mayor significado encontramos la irrupción del cine, las competencias de otros teatros como el Capitolio donde tenían lugar representaciones de mayor envergadura interpretativa y técnica, pero, sobre todo, la aparición del Teatro Principal, construido en 1927 con enormes pretensiones escénicas, convirtiéndose en uno de los mejores teatros de la zona oriental de la isla.

Aunque durante muchos años funcionó como sala de cine, en la actualidad pertenece al Consejo Provincial de las Artes Escénicas y sede de la compañía de danza Osokpuán Irabbo.

Es nuestro deber agradecer a dońa Aracelis Griñán Vazquez, directora de los «Fondos Raros» de la Hemeroteca Provincial de Ciego de Ávila, su generosidad y atenciones; también al personal del Archivo Histórico Provincial «José Gómez Cardoso", y a todas aquellas personas que amablemente facilitaron datos e informaciones acerca de las actividades teatrales en la citada ciudad. Finalmente, a dońa Catherine Täger Pérez por su desinteresada colaboración.

Recibido: mayo de 2019. Aceptado: junio de 2019

22 Idem: Alfonso Fernández, Adalberto: op. cit., p. 207 
